\title{
Clinical Features, Pathological Findings and Treatment of Recurrent Dermatofibrosarcoma Protuberans
}

\author{
Yanan $\mathrm{Li}^{1}$, Chuan Wang${ }^{1}$, Bo Xiang ${ }^{1}$, Siyuan Chen ${ }^{2 凶}, \mathrm{Li} \mathrm{Li}^{3}, \mathrm{Yi} \mathrm{Ji}^{1 凶}$ \\ 1. Division of Oncology, Department of Pediatric Surgery, West China Hospital of Sichuan University, Chengdu, 610041, China; \\ 2. Pediatric Intensive Care Unit, Department of Critical Care Medicine, West China Hospital of Sichuan University, Chengdu, 610041, China; \\ 3. Laboratory of Pathology, West China Hospital of Sichuan University, Chengdu, 610041, China. \\ $\triangle$ Corresponding authors: Siyuan Chen MD, PhD, Pediatric Intensive Care Unit, Department of Critical Care Medicine; Yi Ji MD, PhD, Division of Oncology, \\ Department of Pediatric Surgery, West China Hospital of Sichuan University. \#37 Guo-Xue-Xiang, Chengdu, 610041, China. Tel: +86 28 85422456; Fax: +86 28 \\ 85423453; E-mail: siy_chen@163.com (SYC); jijiyuanyuan@163.com (YJ). \\ (c) Ivyspring International Publisher. This is an open access article distributed under the terms of the Creative Commons Attribution (CC BY-NC) license \\ (https://creativecommons.org/licenses/by-nc/4.0/). See http://ivyspring.com/terms for full terms and conditions.
}

Received: 2016.10.18; Accepted: 2017.03.27; Published: 2017.05.12

\begin{abstract}
Dermatofibrosarcoma protuberans (DESP) is a relatively rare and low-grade tumor of the skin and soft tissue. We review the clinical features, pathological findings and management of recurrent DESP with the aim to improve our understanding of this rare tumor. Fifty-seven patients were diagnosed with recurrent DFSP. The mean age at the presentation of DFSP was 30.9 years. The mean age at diagnosis of DFSP was 40.2 years. Chest wall was the dominant location. The histopathological diagnosis was ordinary DFSP (O-DFSP) in 46 cases and fibrosarcomatous DFSP (FS-DFSP) in 11 cases. No differences were noted between FS-DFSP and conventional DFSP in age at presentation and diagnosis, tumor size, as well as CD34 and SMA expression $(P>0.05)$. However, the time course to recurrence in FS-DFSP group was less than that in O-DFSP group ( $P$ $=0.038$ ). All of the patients were treated with Mohs micrographic surgery (MMS). On follow-up, none of the patients had tumor recurrence. Our data demonstrated that the clinical features of recurrent DFSP are non-specific and variable. Our current practice suggests that MMS is very useful treatment choice for recurrent DFSP.
\end{abstract}

Key words: Dermatofibrosarcoma protuberans; Recurrence; Clinical features; Mohs micrographic surgery.

\section{Introduction}

Dermatofibrosarcoma protuberans (DFSP) is a relatively rare and low-grade sarcoma of the skin and soft tissue. It is reported that the incidence of DFSP is estimated to be 0.8 to 5 cases per million per year and $0.1 \%$ of all malignancies. It approximately constitutes $4 \%$ of all soft tissue sarcomas and usually appears on the trunk and extremities. DFSP presents more commonly in adults between 20 and 50 years of age. The male-to-female ratio is roughly 1:1 (1). DESP has a low potential of metastasis and a high rate of local recurrence. Five-year relative survival rates are high $(2,3)$.

The pathogenesis of DESP is controversial and not completely understood. Molecular studies revealed that chromosomal translocation plays an important pathogenic role in this tumor. The chromosomal translocation is found in more than $90 \%$ of cases, and involves $17 \mathrm{q} 22$ and 22q13 (4). The translocation fuses the genes COL1A1 with the platelet-derived growth factor beta (PDGF $\beta$ ), usually with a ring chromosome formation. This gene codes for a fusion protein, which binds to the constitutively expressed PDGF $\beta$ receptor and acts as an autocrine factor to stimulate the growth of DFSP cells (5). This finding contributes to the development of new diagnostic tools and new treatment strategies. Histologically, DFSP commonly consists of a proliferation of spindle cells in storiform, cartwheel, sheetlike or fascicular pattern. The majority of DFSP lesions were positive for CD34 but negative for S100 and desmin (6).

Previously, the therapeutic regimen for DFSP is 
complete surgical excision with a clear and wide margin. The recurrence rate rages from $0 \%$ to $41 \%$ in cases treated by wide local excision (WLE) with at least 2-3 cm of free margin (7). Mohs micrographic surgery (MMS) has been encouraged in recent decades. The recurrence rates have decreased to $0.6-6 \%(8)$. However, DFSP is often misdiagnosed as a benign tumor and thus is excised by a simple excision, which leads to a high local recurrence rate about $26-60 \%$ (9).

In the present study, we retrospectively evaluated and analyzed all patients with a diagnosis of recurrent DFSP in our institutes over a period of 6 years, with the aim to improve our understanding of recurrent DFSP and prevent tumor recurrence and morbidity.

\section{Methods}

This study was undertaken at the West China Hospital of Sichuan University with the approval of the human ethics review committee. We conducted a retrospective analysis of all patients with recurrent DFSP diagnosed from 2010 to 2016. Written informed consent was obtained from all patients or patients' parents for their clinical data to be used. All patients were admitted into our hospital owing to recurrence of DFSP after a previous surgery performed elsewhere. Recurrent DFSP was defined as tumor regrowth at the original tumor site at least 6 months after the resection. Clinical records were independently reviewed by two investigators. Clinical information, including demographics, clinical presentation, laboratory results, treatment, histopathological findings, follow-up examinations and outcome, was obtained. In all cases, diagnosis was independently confirmed by two expert pathologists.

Based on the histopathological findings, we divided DFSP into 2 types: fibrosarcomatous DFSP (FS-DFSP) and ordinary DFSP (O-DFSP) (10). Statistical analyses of the study were conducted using SPSS 21.0 for Windows (SPSS Inc, Chicago, USA). Student's $t$-test was used to analyze the quantitative data. The Fisher exact test was used to compare qualitative variables. $P$ values less than 0.05 were considered significant.

\section{Results}

Fifty-seven patients with a diagnosis of recurrent DFSP were included for analysis. All of these patients had simple excision at other institutions, and all of them demonstrated local recurrence after resection. The patients were admitted to our hospital after the last recurrence. The main characteristics of patients and the DFSP are summarized in Table 1 . There were
26 females and 31 males, with a female-to-male ratio of $0.84: 1$. The mean age at the presentation of DFSP was 30.9 years (range 3 to 63 years). The mean age at diagnosis of DFSP was 40.2 years (range 4.8 to 76.5 years). The mean interval between the presentation and the diagnosis of the DFSP was 9.0 years. Three patients were younger than 20 years $(5.3 \%)$. Fifty patients were between 20 and 59 years (87.7\%). Four patients were older than 59 years $(7.0 \%)$. Fourteen patients underwent resection more than once because of recurrences. Two patients received adjuvant radiotherapy after resection. The mean time to the first recurrence after the primary simple excision was 2.93 years (range 0.5 to 18 years). The most common symptom of DFSP was present as a painless plaque that protrudes above the surface of the skin at the original tumor site. The most common anatomical location of the lesions was chest wall, representing $29.8 \%$ of all recurrent DFSP, followed by the back, abdominal wall, and head-face-neck area (Figure 1). In the majority of patients (45 patients), the recurrent lesions remained stable during the first several months but grew rapidly later. In contrast, the recurrent lesions in the remaining 12 patients did not grow fast. The lesions in 15 cases were tender on pressure. Five patients had multiple lesions around the scar. Tumor ulceration was observed in 2 patients. The mean size of the lesions was $10.9 \mathrm{~cm}^{2}$ (range 0.3 to $100 \mathrm{~cm}^{2}$ ). Only two patients complained itching of the lesions. In one patient, the onset of lesion was preceded by antecedent trauma.

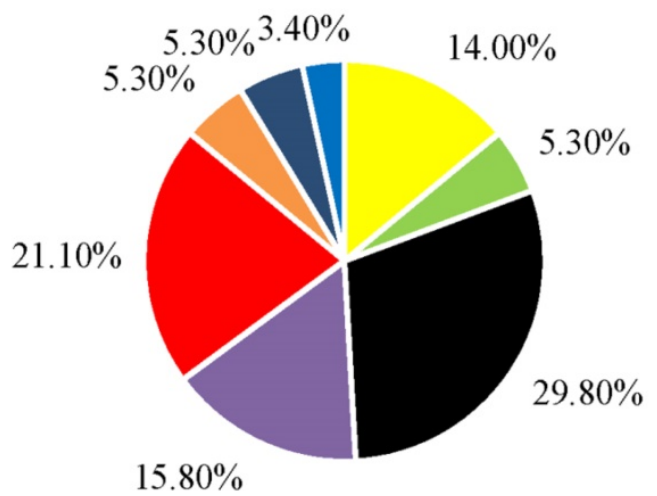

$\begin{array}{lll}\text { " Head-face-neck } & \text { "Shoulde } & \text { - Chest } \\ \text { - Abdomen } & \text { - Back } & \text { " Buttocks } \\ \text { - Upper extremity } & \text { " lower extremity } & \end{array}$

Figure 1. Location of the recurrent dermatofibrosarcoma protuberans

None of the patients had distant metastasis. All of the recurrent cases were treated by MMS using repeat frozen tissue biopsies at our institution. Hematoxylin-eosin staining showed that 46 cases 
were O-DFSP (DFSP without fibrosarcomatous changes). Fibrosarcomatous changes were observed in 11 cases (Figure 2). No differences were noted between FS-DFSP and O-DFSP in age at presentation and diagnosis, gender, and tumor size $(P>0.05)$. The average time to the first recurrence of FS-DFSP was 1.87 years after the primary simple excision, compared with 3.27 years in O-DFSP $(P=0.038)$. Immunohistochemical examination showed that the lesions in 55 patients were positive for CD34. The remaining two lesions were negative for CD34: one each for O-DFSP and FS-DFSP. All of the lesions were positive for Ki-67. The mean Ki-67 index was $14 \%$. Three tumors were positive for SMA and one lesion was positive for S-100. All of the lesions were negative for desmin. There was no significant difference in the positive rate in CD34 and SMA expression between FS-DFSP and O-DFSP $(P>0.05)$ (Table 1).

Table 1. Clinic characteristics of recurrent O-DFSP and recurrent FS-DFSP

\begin{tabular}{|c|c|c|c|c|}
\hline Characteristics & $\begin{array}{l}\text { FS-DFSP } \\
\mathbf{n}=\mathbf{1 1}\end{array}$ & $\begin{array}{l}\text { O-DFSP } \\
\mathrm{n}=46\end{array}$ & $\begin{array}{l}\text { Total } \\
\mathrm{n}=\mathbf{5 7}\end{array}$ & $P$-values \\
\hline \multicolumn{5}{|l|}{ Patients } \\
\hline Age at presentation (year) $*, \dagger$ & $34.6(13.2)$ & $30.0(15.6)$ & $30.9(15.2)$ & 0.374 \\
\hline Age at diagnosis (year) ${ }^{\dagger}$ & $42.5(8.1)$ & $39.7(14.3)$ & $40.2(13.3)$ & 0.532 \\
\hline Mean time to recurrence (year) ${ }^{\dagger}$ & $1.87(1.3)$ & $3.27(4.3)$ & $2.93(3.81)$ & 0.038 \\
\hline Gender $\ddagger$ & & & & 0.738 \\
\hline Female & $4(36.7)$ & $22(47.8)$ & $26(45.6)$ & \\
\hline Male & $7(63.3)$ & $24(52.2)$ & $31(54.4)$ & \\
\hline Number of resections ${ }^{\ddagger}$ & & & & 0.105 \\
\hline 1 & $8(72.7)$ & $35(76.1)$ & $43(75.4)$ & \\
\hline 2 & $1(9.1)$ & $10(21.7)$ & $11(19.3)$ & \\
\hline 3 & $1(9.1)$ & $1(2.2)$ & $2(3.5)$ & \\
\hline 4 & $1(9.1)$ & $0(0.0)$ & $1(1.8)$ & \\
\hline \multicolumn{5}{|l|}{ DFSP } \\
\hline $\operatorname{Size}\left(\mathrm{cm}^{2}\right)^{\dagger}$ & $12.7(17.7)$ & $10.6(19.2)$ & $10.9(18.8)$ & 0.732 \\
\hline \multicolumn{5}{|l|}{ Immunohistochemical examination $\ddagger$} \\
\hline CD34 & & & & 0.352 \\
\hline Negative & $1(9.1)$ & $1(2.2)$ & $2(3.5)$ & \\
\hline Positive & $10(90.9)$ & $45(97.8)$ & $55(96.5)$ & \\
\hline SMA $\ddagger$ & & & & 0.384 \\
\hline Negative & $11(100.0)$ & $43(93.5)$ & $54(94.7)$ & \\
\hline Positive & $0(0.0)$ & $3(6.5)$ & $3(5.3)$ & \\
\hline Ki-67 & & & & $\mathrm{N} / \mathrm{A}$ \\
\hline Negative & $0(0.0)$ & $0(0.0)$ & $0(0.0)$ & \\
\hline Positive & $11(100.0)$ & $46(100.0)$ & $57(100.0)$ & \\
\hline Desmin & & & & $\mathrm{N} / \mathrm{A}$ \\
\hline Negative & $0(0.0)$ & $0(0.0)$ & $0(0.0)$ & \\
\hline Positive & $11(100.0)$ & $46(100.0)$ & $57(100.0)$ & \\
\hline Follow-up (years) ${ }^{\dagger}$ & $2.18(1.3)$ & $3.29(1.6)$ & 3.07 (1.6) & 0.028 \\
\hline
\end{tabular}

*Age at diagnosis was defined as the date Dermatofibrosarcoma protuberans was first confirmed by pathology.

tData given as mean (SD).

$\ddagger$ Values are presented as number (percentage).

O-DFSP: Ordinary dermatofibrosarcoma protuberans; FS-DFSP: Fibrosarcomatous dermatofibrosarcoma protuberans

A)

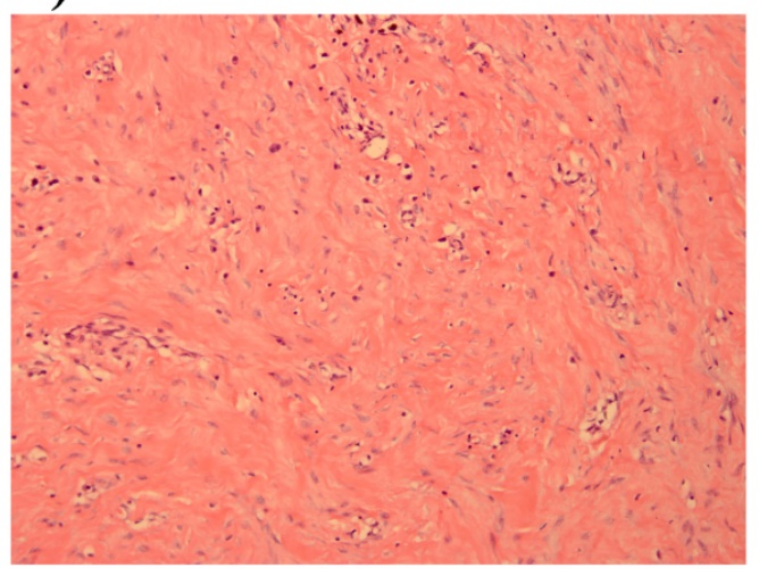

B)

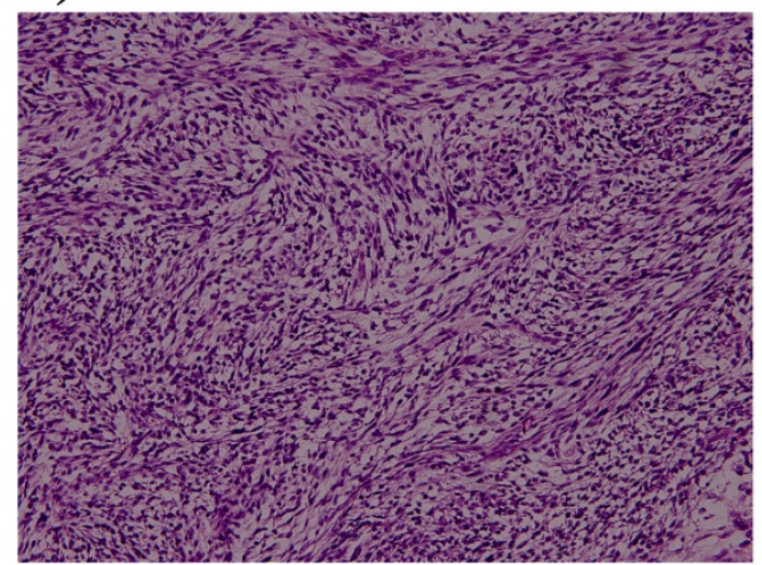

Figure 2. Histopathological examination A) DFSP without fibrosarcomatous change showing spindle cells (H\&E, original magnification $\times 200)$. B) DFSP with fibrosarcomatous changes showing a fascicular growth pattern $(\mathrm{H} \& \mathrm{E}$, original magnification $\times 200)$. 
The mean length of follow-up after the MMS for the entire cohort was 3.07 years (range 0.5 to 6 years). All the patients were followed up every 3 months for the first 1 year and then every 6 months thereafter. Clinical exam, local ultrasound (US) or magnetic resonance imaging (MRI), along with a chest $X$-ray or chest computer tomography (CT) were the modalities used to monitor patients. All of the patients had a favorable outcome with no tumor recurrence and metastasis during follow-up.

\section{Discussion}

Previously, a small number of case reports described the characteristics of recurrent DFSP. However, the findings were based on small sample sizes. To identify the characteristics of recurrent DFSP, more extensive studies are required. In the current study, we successfully provided further insights into the clinical features, pathological characteristics and treatment of recurrent DFSP. To the best of our knowledge, this is the largest reported series of recurrent DFSP.

DFSP can present in any part of the body. In a recent large retrospective study, the authors found that the most common site in primary cases is the trunk, followed by extremities. Head-face-neck areas are less common (11). In the current study, we found that recurrent cases more common had tumors of the head-face-neck areas than of the extremities. Lesions of the head-face-neck were more likely to present challenges for surgeons to achieve adequate margins than lesions of extremities because of their functionally and cosmetically sensitive anatomic locations. Primary DFSP commonly grew slowly, ranging from several months to many years (12). In our study, the majority of recurrent lesions also grew slowly at the beginning but on subsequent grew rapidly. $\mathrm{K}$ et al. reported that patients with large lesions have a high possibility to be associated with FS-DFSP (13). Interestingly, in the present study, there was no significant difference in tumor size between recurrent FS-DFSP and recurrent O-DFSP. The primary tumor is commonly solitary. In the current study, 5 cases showed multiple nodules $(8.8 \%)$. These data suggest that recurrent DFSP might have some growth characteristics similar to that of primary DFSP, but they also display some degree of unique growth pattern.

Occasionally, DFSP may exhibit high-grade histopathological features, when it is then termed FS-DFSP. FS-DFSP typically appears as an abrupt or gradual transition into cell-rich spindle-cell fascicles with cytological atypia and increased mitotic figure rate (14). In the majority of cases, immunostaining of DFSP shows positivity for CD34. Therefore, CD34 is a highly recommended DFSP tumor marker for immunohistologic analysis (6). It is reported that FS-DFSP may show loss of CD34 expression in about half of the cases (15). In the current study, however, we found that only one case of FS-DFSP (9.1\%) was negative for CD34. While the reason for this is not known. In addition to CD34, recent studies demonstrated that the analyses of PDGF $\beta$ and nestin expression were also effective for pathological diagnosis of DFSP $(16,17)$. Unfortunately, none of these markers are specific and are not sufficient to distinguish DFSP from other types of cutaneous tumors as alone.

MMS is important in tumors with poorly defined margins, particularly in lesions on the head-face-neck areas and acral regions, and in the treatment of recurrent and pediatric patients. Compared with wide local excision, MMS has the advantage of conserving tissue, causing a smaller wound size. Furthermore, the possibility of recurrence can be decreased (8). The resection of recurrent DFSP is more difficult, because the tumor is very likely to violate deeper tissues. In present study, none of the patients who had MMS recurred during follow-up. However, MMS can be time consuming, and be difficult to perform on large lesions. In addition, although data in recurrent cases are lacking, data in primary cases found no difference in overall survival for patients treated with wide local excision versus MMS (11).

Advances in the understanding of the molecular mechanisms of DFSP have applied in clinical practice via targeted therapy acting on PDGFR. The first effective systemic therapy in DFSP introduced into clinical practice was imatinib mesylate. It is demonstrated usefully for recurrent, metastatic or inoperable tumors. In order to facilitate the removal of large tumor, imatinib can also be used preoperatively to shrink the tumor size (18). However, the response rate to imatinib was only $46 \%$, and in FS-DFSP, the responses are even weaker and less durable. Tumor relapse might occur once imatinib treatment is terminated. A recent preclinical study suggested that a combination regimen, including immunotherapy, might be beneficial in certain cases (21). Remarkably, sunitinib, a multi-targeted receptor tyrosine kinase inhibitor, is currently being tested in a prospective clinical trial for diagnoses that include, but are not limited to, metastasis and recurrent DFSP (ClnicalTrials.gov identifier NCT00474994).

In a recent systematic review, the authors found that the mean time to recurrence was 5.7 years after MMS (8). In the present study, we found that the mean time to recurrence was 2.93 years after simple excision. Interestingly, we found the mean time to recurrence of FS-DFSP was shorter than that of 
O-DFSP. Multiple studies have reported that FS-DFSP was more aggressive form of DFSP than O-DFSP (20, 22). This difference may explain why FS-DFSP presented a shorter time to recurrence. Although none of our patient had local recurrence and metastases after being treated with MMS, there is insufficient data, at our institution and in the literature, in reporting long-term follow-up data in order to ensure the efficacy of MMS in recurrent DFSP. Therefore, further efforts are needed to evaluate the definite effect of MMS on recurrent cases.

\section{Conclusion}

This report is the largest study assessing the clinical features, pathological characteristics and treatment of recurrent DFSP. The data presented here demonstrate that the clinical characteristics of recurrent DFSP are non-specific and variable. Metastases are rare in these patients. The cases with short time to recurrence were more likely to be FS-DFSP. The main choice for recurrent DFSP is complete surgical excision. Although long-term follow-up data are lacking, our current practice suggests that MMS is very useful for recurrent cases. Due to its high local recurrence rate, clinical follow-up is thus advised.

\section{Acknowledgements}

This project was supported by grants from the National Natural Science Foundation of China (Grants 81401606 and 81400862) and the Science Foundation for The Excellent Youth Scholars of Sichuan University (2015SU04A15). The authors are indebted to all editors and reviewers for their kindly reviewing of the manuscript.

\section{Competing Interests}

The authors have declared that no competing interest exists.

\section{References}

1. Kreicher KL, Kurlander DE, Gittleman HR, Barnholtz-Sloan JS, Bordeaux JS. Incidence and survival of primary dermatofibrosarcoma protuberans in the united states. Dermatol Surg 2016; 42 Suppl 1: S24-S31

2. Weinstein JM, Drolet BA, Esterly NB, Rogers M, Bauer BS, Wagner AM et al. Congenital dermatofibrosarcoma protuberans: Variability in presentation. Arch Dermatol 2003; 139: 207-211

3. Saiag P, Grob JJ, Lebbe C, Malvehy J, Del MV, Pehamberger H et al. Diagnosis and treatment of dermatofibrosarcoma protuberans. European consensus-based interdisciplinary guideline. Eur J Cancer 2015; 51: 2604-2608

4. Sirvent N, Maire G, Pedeutour F. Genetics of dermatofibrosarcoma protuberans family of tumors: From ring chromosomes to tyrosine kinase inhibitor treatment. Genes Chromosomes Cancer 2003; 37: 1-19

5. Simon MP, Pedeutour F, Sirvent N, Grosgeorge J, Minoletti F, Coindre JM et al. Deregulation of the platelet-derived growth factor B-chain gene via fusion with collagen gene COL1A1 in dermatofibrosarcoma protuberans and giant-cell fibroblastoma. Nat Genet 1997; 15: 95-98

6. Abenoza P, Lillemoe T. CD34 and factor XIIIa in the differential diagnosis of dermatofibroma and dermatofibrosarcoma protuberans. Am J Dermatopathol 1993; 15: 429-434

7. Valdivielso-Ramos M, Hernanz JM. Dermatofibrosarcoma protuberans in childhood. Actas Dermosifiliogr 2012; 103: 863-873
8. Foroozan M, Sei JF, Amini M, Beauchet A, Saiag P. Efficacy of Mohs micrographic surgery for the treatment of dermatofibrosarcoma protuberans: Systematic review. Arch Dermatol 2012; 148: 1055-1063

9. Jia J, Zheng Y, Dong X, Wang X, Yang J, Ying Z et al. Dermatofibrosarcoma protuberans with pit-like lesions: A case report and literature review. Oncol Lett 2015; 10: 3765-3768

10. Stacchiotti S, Astolfi A, Gronchi A, Fontana A, Pantaleo MA, Negri T et al. Evolution of dermatofibrosarcoma protuberans to DFSP-Derived fibrosarcoma: An event marked by Epithelial-Mesenchymal transition-like process and 22q loss. MOL Cancer Res 2016; 14: 820-829

11. Criscito MC, Martires KJ, Stein JA. Prognostic factors, treatment, and survival in dermatofibrosarcoma protuberans. JAMA Dermatol 2016; [Epub ahead of print].

12. Zhang L, Liu OY, Cao Y, Zhong JS, Zhang WD. Dermatofibrosarcoma protuberans: Computed tomography and magnetic resonance imaging findings. Medicine (Baltimore) 2015; 94: e1001

13. Hayakawa K, Matsumoto S, Ae K, Tanizawa T, Gokita T, Funauchi Y et al. Risk factors for distant metastasis of dermatofibrosarcoma protuberans. J Orthop Traumatol 2016; 17: 261-266

14. Hoesly PM, Lowe GC, Lohse CM, Brewer JD, Lehman JS. Prognostic impact of fibrosarcomatous transformation in dermatofibrosarcoma protuberans: A cohort study. J Am Acad Dermatol 2015; 72: 419-425

15. Mentzel T, Beham A, Katenkamp D, Dei TA, Fletcher CD. Fibrosarcomatous ("high-grade") dermatofibrosarcoma protuberans: Clinicopathologic and immunohistochemical study of a series of 41 cases with emphasis on prognostic significance. Am J Surg PathoL 1998; 22: 576-587

16. Nakamura I, Kariya Y, Okada E, Yasuda M, Matori S, Ishikawa O et al. A novel chromosomal translocation associated with COL1A2-PDGFB gene fusion in dermatofibrosarcoma protuberans: PDGF expression as a new diagnostic tool. JAMA Dermatol 2015; 151: 1330-1337

17. Sellheyer K, Nelson P, Krahl D. Dermatofibrosarcoma protuberans: A tumour of nestin-positive cutaneous mesenchymal stem cells? Br J Dermatol 2009; 161: 1317-1322

18. Wang C, Luo Z, Chen J, Zheng B, Zhang R, Chen Y et al. Target therapy of unresectable or metastatic dermatofibrosarcoma protuberans with imatinib mesylate: An analysis on 22 Chinese patients. Medicine (Baltimore) 2015; 94: e773

19. Stacchiotti S, Pantaleo MA, Negri T, Astolfi A, Tazzari M, Dagrada GP et al. Efficacy and biological activity of imatinib in metastatic dermatofibrosarcoma protuberans (DFSP). Clin Cancer Res 2016; 22: 837-846

20. Stacchiotti S, Pedeutour F, Negri T, Conca E, Marrari A, Palassini E et al. Dermatofibrosarcoma protuberans-derived fibrosarcoma: Clinical history, biological profile and sensitivity to imatinib. Int J Cancer 2011; 129: 1761-1772

21. Tazzari M, Indio V, Vergani B, De Cecco L, Rini F, Negri T et al. Adaptive immunity in fibrosarcomatous dermatofibrosarcoma protuberans and response to imatinib treatment. J Invest DermatoL 2016; [Epub ahead of print].

22. Abbott JJ, Oliveira AM, Nascimento AG. The prognostic significance of fibrosarcomatous transformation in dermatofibrosarcoma protuberans. Am J Surg Pathol 2006; 30: 436-443 